\title{
Renal Replacement Therapy for Patients Requiring Extracorporeal Membrane Oxygenation: A Multicenter International Survey
}

Michael THY ( $\sim$ michael245thy@gmail.com )

Assistance Publique - Hopitaux de Paris https://orcid.org/0000-0001-9366-505X

Pascal Augustin

AP-HP: Assistance Publique - Hopitaux de Paris

Alexy Tran-Dinh

AP-HP: Assistance Publique - Hopitaux de Paris

Philippe Montravers

AP-HP: Assistance Publique - Hopitaux de Paris

Christian de Tymowski

AP-HP: Assistance Publique - Hopitaux de Paris

\section{Research}

Keywords: Acute kidney injury (AKI), extracorporeal membrane oxygenation (ECMO), renal replacement therapy (RRT), dialysis catheter (DC), PubMed database

Posted Date: June 15th, 2021

DOl: https://doi.org/10.21203/rs.3.rs-592881/v1

License: (c) (i) This work is licensed under a Creative Commons Attribution 4.0 International License.

Read Full License

Version of Record: A version of this preprint was published at Blood Purification on April 7th, 2022. See the published version at https://doi.org/10.1159/000522398. 


\section{Abstract}

\section{Objectives}

Patients receiving extracorporeal membrane oxygenation (ECMO) often require renal replacement therapy (RRT). Insertion of a dialysis catheter may be challenging in these patients. Direct connection of RRT lines on an ECMO circuit (DCRE) may help circumvent this problem. However, no guidelines exist on this issue, and various types of practices may exist. This study aimed to describe the practice of RTT in patients on ECMO, including the direct connection of RTT lines on the ECMO circuit (DCRE) as well as the perception and concerns related to this technique.

\section{Design}

International survey

\section{Setting.}

Worldwide diffusion via email.

\section{Subject.}

Professionals involved in the management of ECMO

\section{Interventions:}

Respondents' characteristics were analyzed. Respondents always or often performing RRT via the ECMO circuit were classified in the ECMO group, and those using a dialysis catheter were classified in the DC group. The two groups were compared regarding their characteristics and their practice in ECMO and RRT. Participants were asked about their perceptions of DCRE and actual problems previously encountered.

\section{Measurements and Main Results:}

From March 2019 to October 2019, 298 participants answered the questionnaire from 46 different countries. Only $28 \%$ were working in pediatric departments. Among the 165 participants commonly performing RRT in patients on ECMO, 100 (61\%) performed mainly RRT via the ECMO circuit, and 65 (39\%) performed RRT via DC. Pediatric practice and a longer experience were the only noticeable characteristics of the ECMO group. The most reported fear regarding DCRE was the risk of air embolism $(n=84,28 \%)$, but the most encountered problem was unmanageable pressure alarms in RRT devices.

\section{Conclusion}

The present study showed significant heterogeneity in RRT practices in patients on ECMO. The lower experience of the DC group, the high rates of fears toward DCRE, and pressure alarm issues suggested that protocols and training may overcome reluctance and technical difficulties. Further research may provide data to back specific guidelines. 


\section{Take-home message}

The present study showed significant heterogeneity in RRT practices in patients on ECMO showing lower experience, fears and pressure alarm issues as barriers for direct connection of RRT lines on ECMO circuit, suggesting needs for protocols, training and specific guidelines.

\section{0-character Tweet}

Low experience, fears and pressure alarm issues as barriers for direct connection of RRT lines on ECMO circuit, suggesting needs for protocols, training and specific guidelines.

\section{Introduction}

Acute kidney injury (AKI) is a frequent condition in patients requiring extracorporeal membrane oxygenation (ECMO). Up to $70 \%(1-3)$ of adult patients and $60 \%$ of children requiring ECMO need renal replacement therapy (RRT) (4). An efficient RRT procedure is required to manage metabolic disorders and fluid overload $(3,5-9)$. Vascular access for dialysis catheter (DC) insertion may not be easy in patients on ECMO as the ECMO circuit often occupies two vascular access sites. Insertion of a DC may be even more challenging in these patients receiving anticoagulation therapy and/or with coagulation disorders. Furthermore, DC may be associated with blood flow limitations and dysfunction, especially in left jugular and subclavian sites $(10,11)$, and adverse events $(12,13,14)$. Direct connection of a device to the ECMO circuit (DCRE) may circumvent these problems and may allow higher blood flow (15). Some studies have shown that DCRE is feasible and may increase RRT circuit life expectancy $(16,17)$. Nevertheless, this approach remains poorly described and may be associated with high pressures in the RRT circuit responsible for iterative interruptions of RRT sessions, loss of hemofilters, and inefficiency $(18,19)$. Moreover, this technique requires manipulating ECMO circuits and exposes patients to a theoretical risk of air embolism, pumping stop, or blood loss.

In classical RRT, the main determinants of inadequate pressures and hemofilter clotting are related to the settings of RRT, including blood flow, volume replacement therapy, and the resulting filtration fraction as well as the type (e.g., citrate vs. heparin) and level of anticoagulation (20). In DCRE, the choice of connection locations can be challenging (21) because of high pressures in the different segments of the ECMO circuit. ECMO pressures mainly depend on ECMO cannula size, ECMO blood flow, and ECMO oxygenation membrane characteristics (22-24). Specific protocols may help perform this technique more easily and safely (25). However, to date, no guidelines have been published to help physicians perform RRT in patients placed on ECMO, and a wide range of practices may exist. We hypothesized that DCRE is not overwhelmingly used and that its perceived level of difficulty may greatly vary.

The aim of our study was to assess existing practices of RRT in patients requiring ECMO and to identify factors associated with DCRE practice. Furthermore, we aimed to collect physician opinions and their fears regarding DCRE. 


\section{Material And Methods \\ Study design}

This was an international cross-sectional study based on an online questionnaire targeting health care professionals involved in the management of ECMO. Centers managing ECMO were identified on the internet using affiliations of articles related to this topic published within 5 years $(n=665)$. These centers and affiliated authors were searched using the PubMed database, and authors (PA, MT, and CDT) collected data.

\section{Questionnaire building}

The questionnaire was available on a general data protection regulation-compliant electronic survey using Surveymonkey ${ }^{\circledR}$ web-based application. Survey questions were written in English and were distributed for comments using a modified Delphi technique to all members of the research group. After several revisions, the final questionnaire was approved and driven by all authors. The questionnaire was registered at the French Data Protection Authority (Commission Nationale de I'Informatique et des Libertés - CNIL).

\section{Survey description}

The web-based survey needed approximately between 15 and 20 minutes to be completed after information about the survey, and it was divided into the following four sections: main respondents' characteristics, usual management of RRT, usual management of ECMO, and management of RRT in patients requiring ECMO. Questions assessing the frequencies of practices were assessed by 4-point Likert scale multiple-choice questions, labeled "Never", "Rarely", "Often", and "Always". To explore the perceived difficulty of performing DCRE, we used a visual analog slide scale ranging from 0 to $100(0$, No difficulty; and 100, Impossible to perform).

All questions covered key issues previously raised in the existing literature, including articles published by authors $(16,21)$. Usual management of RRT refers to the way classical RRT is usually performed in each institution.

The classification of income level of countries was based on the World Bank database (26).

\section{Data analysis}

Respondents were classified as those working in adult departments and those working in pediatric departments. Likert data were analyzed by dichotomizing answers in two classes (negative answer: Never/Rarely vs. positive answer: Often/Always). The sum of the positive answers (often/always) could be superior or inferior to $100 \%$. To assess characteristics associated with the use of DCRE, we compared the respondents who "Always" or "Often" performed DCRE (called the ECMO group) to those who "Always" or "Often" performed RRT via DCs (called the DC group). Therefore, participants who 
never/rarely performed RRT via DCs and never/rarely performed RRT via DCRE were excluded from this analysis.

The difficulty rating of DCRE was categorized as "easy" and "difficult" with a cutoff of 50/100 corresponding to the third interquartile of global rating.

\section{Statistical Analysis}

The results are expressed as the median and interquartile range (IQR [25-75\%]) for continuous variables, and the results are expressed as numbers and percentages for categorical variables. Categorical data were analyzed using the Chi-square or Fisher exact test when appropriate. Continuous data were compared using the Mann-Whitney $U$ test. All reported statistical tests were 2-sided, and $p$ values $<0.05$ were considered statistically significant. Statistical analysis was performed by $\mathrm{R} \circledast 3.4 .2$ software.

\section{Results}

Overview of respondents' characteristics and usual RRT practices.

From March 2019 to July 2019, 298 participants from 46 countries answered the questionnaire. The main characteristics of the respondents are shown in Table 1. Supplemental Fig. 1 shows the distribution of respondents' countries. The two main respondents' countries were France and the United States of America. Half of the respondents worked in departments managing less than 3 new patients on ECMO each month, and $37 \%$ managed ECMO for more than 10 years. The predominant usual RRT types were techniques using continuous modalities of RRT, and $62 \%$ of respondents declared often using femoral DCs. Slightly more than half of respondents reported citrate as the main anticoagulation in their usual practice of RRT. 
Table 1

Main characteristics of respondents

\begin{tabular}{|c|c|}
\hline Characteristics & $\begin{array}{l}\text { Overall } \\
\text { (n:298) }\end{array}$ \\
\hline \multicolumn{2}{|l|}{ Continent } \\
\hline Europe & $141(47)$ \\
\hline North America & $87(29)$ \\
\hline Asia & $35(12)$ \\
\hline South America & $23(8)$ \\
\hline Oceania & $7(2)$ \\
\hline Africa & $2(1)$ \\
\hline High income country & $249(84)$ \\
\hline \multicolumn{2}{|l|}{ Type of critical care unit } \\
\hline Cardiac surgery ICU & $110(37)$ \\
\hline Polyvalent ICU & $74(25)$ \\
\hline Medical ICU & $44(15)$ \\
\hline Surgical ICU & $36(12)$ \\
\hline Trauma center & $17(6)$ \\
\hline Others & $19(6)$ \\
\hline \multicolumn{2}{|l|}{ Medical specialty } \\
\hline Medical intensivist & $101(34)$ \\
\hline Anesthesiologist-Intensivist & $97(33)$ \\
\hline Medical physician & $12(4)$ \\
\hline Pediatrician & $34(11)$ \\
\hline Surgeon & $32(11)$ \\
\hline \multicolumn{2}{|c|}{ Number of new ECMO cases per month } \\
\hline $0-3$ & $150(50)$ \\
\hline $4-5$ & $65(22)$ \\
\hline $6-10$ & $32(11)$ \\
\hline $11-20$ & $21(7)$ \\
\hline
\end{tabular}




\begin{tabular}{|ll|}
\hline Characteristics & $\begin{array}{l}\text { Overall } \\
\text { (n:298) }\end{array}$ \\
\hline$>20$ & $28(9)$ \\
\hline Personal ECMO experience & \\
\hline$<1$ year & $7(2)$ \\
\hline $1-5$ years & $46(15)$ \\
\hline $5-10$ years & $62(21)$ \\
\hline$>10$ years & $100(34)$ \\
\hline
\end{tabular}

\section{Approach of RRT in patients placed on ECMO}

In patients requiring RRT and ECMO, only 165 participants always or often practiced RRT either via the ECMO circuit or via DC. The ECMO group comprised 100 (61\%) participants, and the DC group comprised 65 (39\%) participants. Other participants reported never or rarely performing RRT via either DC or DCRE (n $=81)$. The remaining 52 participants did not answer this question.

Table 2 summarizes problems previously encountered during the DCRE procedure and fears linked to it. Forty-four percent of respondents reported fears, while 33\% had already encountered problems. The most reported current problem and the most reported fear were not similar (unmanageable pressure alarms and air embolism, respectively). 
Table 2

Main fears and actual encountered problems with the DCRE procedure

\begin{tabular}{|llll}
\hline Fears & \multicolumn{3}{l}{ Problems already encountered } \\
\hline At least one fear & $130(44)$ & At least one problem & $98(33)$ \\
\hline Unstoppable arterial pressure alarm & $50(17)$ & Unstoppable arterial pressure alarm & $58(20)$ \\
\hline Unstoppable venous pressure alarm & $36(12)$ & Unstoppable venous pressure alarm & $44(15)$ \\
\hline Globally stressful & $35(12)$ & Unstoppable TMP alarm & $23(8)$ \\
\hline Unstoppable TMP alarm & $32(11)$ & Air embolism* & $26(9)$ \\
\hline Blood loss & $30(10)$ & Insufficient RRT blood flow & $15(5)$ \\
\hline & & Significant blood loss & $6(2)$ \\
\hline
\end{tabular}

Categorical variables are expressed as $\mathrm{n}(\%)$.

Definition: TMP = Transmembrane pressure; RRT = Renal replacement therapy.

*Including micro air bubbles in ECMO circuit without clinical consequences.

Reasons given by the participants of the DC group for not performing DCRE are listed in Table 3. The three main reasons were the absence of institutional protocol/guidelines, safety concerns, and unmanageable pressure alarms in the RRT device. Nine participants reported having stopped performing DCRE after having experienced problems.

Table 3

Main reasons for not performing DCRE reported by the DC Group

Reasons $n=65$

No institutional protocol

$31(48)$

No guidelines

DCRE perceived as unsafe

Difficult management of pressure alarms

No $Y$ connectors on ECMO circuit

Perceived as an inefficient technique

Categorical variables are expressed as $\mathrm{n}(\%)$.

Definition: $\mathrm{DCRE}=$ Direct connection of RRT on ECMO; DC = Dialysis catheter; ECMO = Extra corporeal membrane oxygenation; RRT = Renal replacement therapy.

\section{Comparisons between the ECMO group and the DC group}


The comparisons between the ECMO group and the DC group for general characteristics, usual RRT management, and usual ECMO management are shown in Supplemental table 1. Participants in the ECMO group reported a longer center and personal ECMO experience and more systematic $Y$-connectors on ECMO circuits, and these participants were more often pediatric physicians. No significant differences regarding ECMO brand distribution, the size of ECMO cannulas, usual RRT settings, usual anticoagulation monitoring, or the rate of monitoring pressure of ECMO circuit were found (analysis performed only among responses of adult departments for cannula sizes and RRT settings).

\section{Technical management of DCRE}

Table 4 shows the management details of DCRE. Intermittent HD was seldom mentioned as an RRT choice for DCRE. Up to $61 \%$ reported modifying RRT or ECMO settings in case of high-pressure alarms. Figure 1 shows the preferred locations of connection reported by the 100 participants of the ECMO group. Most participants selected a connection of both arterial and venous RRT lines between the ECMO pump and the ECMO membrane. Supplemental table 3 shows the explanations for the choices of connection locations, mainly motivated by safety purposes.

Table 4

Management of DCRE in ECMO group

$n=100$

Individuals who perform the connections

Perfusionists

Nurses

$41(41)$

Type of RRT in case of DCRE (always/often)

CVVHF

CVVHDF

CVVHD

IHD

Choice of RRT type differs when DCRE is performed

Adapting initial RRT settings in case of DCRE

Change RRT or ECMO settings in case of high-pressure alarms

Modify the locations of RRT lines in case of high-pressure alarms $10(10)$

Categorical variables are expressed as numbers and percentages.

Definitions: DCRE = Direct connection of RRT on ECMO; ECMO = Extra corporeal membrane oxygenation; RRT = Renal replacement therapy; $\mathrm{IHD}=$ Intermittent hemodialysis; $\mathrm{CVVHF}=$ Continuous veno-venous hemofiltration; CVVHDF = Continuous veno-venous hemodiafiltration; CVVHD = Continuous veno-venous hemodialysis. 


\section{Difficulty rating of DCRE and training}

The boxplot representing the difficulty of performing DCRE on the analog visual scale from 0 to 100 for the pooled two groups is shown in Supplemental Fig. 2. The median rating was $21[5-50]_{1 \mathrm{QR} 25-75 \%}$, and $55 \%$ of respondents appreciated training sessions on DCRE versus $28 \%$ on RRT and $22 \%$ on ECMO. A comparison between the respondents who considered DCRE to be a difficult procedure and others (rating $\geq 50$ ) is displayed in Supplemental table 1. Personal and center experience in ECMO were the main factors associated with the perceived difficulty of DRCE.

\section{Discussion}

This first survey exploring the practice of RRT in patients on ECMO demonstrates heterogeneity in RRT practices in patients on ECMO. Among the 165 participants commonly performing RRT in patients on ECMO, $61 \%$ reported often practicing DCRE. However, up to $20 \%$ of participants found it difficult to perform RRT in patients on ECMO, and more than $50 \%$ were interested in receiving training on this issue.

DCRE was described early in the pediatric population, in which the challenge of vascular sparing is of utmost importance. As opposed to the adult population, the successful utilization of ECMO in children was defined in the 1970s (27), explaining the added expertise. Not surprisingly, our data confirmed that this technique is more common in pediatric practice.

We believe that factors impacting the life expectancy of the RRT filter and RRT pressures (ECMO cannula sizes, anticoagulation, and RRT settings) may influence the use of DCRE. However, the only relevant factors associated with DCRE practice were pediatric practice and personal/center experience in ECMO. Other expected factors, such as ECMO cannula size, were not associated with the use of DCRE. For example, the use of larger arterial cannulas may help reduce postpump pressure (22), which may decrease the frequency of postpump connection failures caused by high pressure in CVVH access lines. In contrast, the use of smaller arterial cannulas is safer when a percutaneous approach is chosen (28). Small venous cannulas may generate marked negative pressures in the prep ump ECMO line (23) inadequate for RTT connection. However, we did not find differences in ECMO cannula sizes between the ECMO group and the DC group (Supplemental table 1). In fact, CRRT devices are manufactured to be connected to central venous pressure ranging from 0 to $20 \mathrm{~mm} \mathrm{Hg}$. Pressures in the ECMO circuit are negative before the pump (segment 1) and positive between the pump and the oxygenator (segment 2) (Fig. 1) $(17,22,23)$. Extreme pressures may be particularly observed with small cannulas and with high ECMO blood flow $(15,22,23)$.

There are different types of obstacles to using DCRE. There are technical issues with some specific ECMO brands not allowing $Y$ connectors. Furthermore, several authors have suggested that some sites of connection are unsafe and not recommended $(19,29)$. Our data showed that DCRE may cause changes in behavior in RRT practices and settings by some physicians, while others may consider this approach unwise, which may be a source of fear. Protocols and training may be helpful for physicians with low 
experience in ECMO. Indeed, medical education, training and simulation may overcome reluctance and problems, including those related to the management of RRT pressure and safety concerns. Further research to provide evidence and guidelines is necessary to legitimize and promote the use of DCRE.

The present study had several limitations. We excluded a relatively large number of participants who never or rarely performed RRT in patients on ECMO from the ECMO and DC groups. This observation was in accordance with a previous study reporting a $20 \%$ rate of physicians never performing RRT in patients on ECMO (30). There was a limitation based on the language of the questionnaire with potential bias in participants' countries. The most interested participants may have willingly answered the questionnaire, while some participants did not answer all questions. It is possible that the participants most involved in these issues answered more carefully than others. In all surveys, answers may not provide reliable features of reality, especially regarding technical issues.

\section{Conclusion}

Our study demonstrated heterogeneity in the clinical practices of RRT in patients on ECMO with a substantial number of participants not using DCRE. There were discrepancies between participants' fears and actual encountered problems. Even though the methodology failed to demonstrate relevant technical issues limiting the practice of DCRE, the need for training and protocols to circumvent physicians' fears and reluctances is strongly supported by our results. Guidelines should be established to help promote good and safe practices.

\section{Declarations}

- Ethics approval and consent to participate:

Informed consent. No animal or human participants involved. No funding. No conflict of interest.

- Consent for publication and Authors' contributions:

All participants consent for publication, all authors consent for publication and equally contributed to the manuscript

- Authors' contributions :

MT, PA and CDT designed the study and collected the data.

All authors analysed and interpreted the data.

MT, PA and CDT wrote the first version of the manuscript, with contributions from ATD, PM

All authors had full access to all the data in the study and had final responsibility for the decision to submit for publication. 
All authors read and approved the final manuscript.

- Availability of data and material: NA

- Competing interests : No competing interests, no conflict of interest

- Funding : No funding

\section{References}

1. Seczyńska B, Królikowski W, Nowak I, et al. Continuous Renal Replacement Therapy During Extracorporeal Membrane Oxygenation in Patients Treated in Medical Intensive Care Unit: Technical Considerations: CRRT During ECMO-Technical Considerations. Ther Apher Dial. 2014;18:523-34.

2. Cheng R, Hachamovitch R, Kittleson M, et al. Complications of Extracorporeal Membrane Oxygenation for Treatment of Cardiogenic Shock and Cardiac Arrest: A Meta-Analysis of 1,866 Adult Patients. Ann Thorac Surg. 2014;97:610-6.

3. Askenazi DJ, Selewski DT, Paden ML, et al. Renal Replacement Therapy in Critically III Patients Receiving Extracorporeal Membrane Oxygenation. Clin J Am Soc Nephrol. 2012;7:1328-36.

4. Hansrivijit $P$, Lertjitbanjong $P$, Thongprayoon $C$, et al. Acute Kidney Injury in Pediatric Patients on Extracorporeal Membrane Oxygenation: A Systematic Review and Meta-analysis. Medicines. 2019;6:109.

5. Shum H-P, Kwan AM-C, Chan K-C, et al. The Use of Regional Citrate Anticoagulation Continuous Venovenous Hemofiltration in Extracorporeal Membrane Oxygenation. ASAIO J. 2014;60:413-8.

6. Haneya A, Diez C, Philipp A, et al. Impact of Acute Kidney Injury on Outcome in Patients With Severe Acute Respiratory Failure Receiving Extracorporeal Membrane Oxygenation*. Crit Care Med. 2015;43:1898-906.

7. Fleming GM, Askenazi DJ, Bridges BC, et al. A Multicenter International Survey of Renal Supportive Therapy During ECMO: The Kidney Intervention During Extracorporeal Membrane Oxygenation (KIDMO) Group. ASAIO J. 2012;58:407-14.

8. Kielstein JT, Heiden AM, Beutel G, et al. Renal function and survival in 200 patients undergoing ECMO therapy. Nephrol Dial Transplant. 2013;28:86-90.

9. Kilburn DJ, Shekar K, Fraser JF: The Complex Relationship of Extracorporeal Membrane Oxygenation and Acute Kidney Injury: Causation or Association? BioMed Res Int 2016; 2016:1-14.

10. Parienti J-J, Mégarbane B, Fischer M-O, et al. Catheter dysfunction and dialysis performance according to vascular access among 736 critically ill adults requiring renal replacement therapy: A randomized controlled study. Crit Care Med. 2010;38:1118-25.

11. Baldwin I, Bellomo R, Koch B. Blood flow reductions during continuous renal replacement therapy and circuit life. Intensive Care Med. 2004;30:2074-9.

12. Maki DG, Kluger DM, Crnich CJ. The risk of bloodstream infection in adults with different intravascular devices: a systematic review of 200 published prospective studies. Mayo Clin Proc. 
2006;81:1159-71.

13. Merrer J, De Jonghe B, Golliot F, et al. Complications of femoral and subclavian venous catheterization in critically ill patients: a randomized controlled trial. JAMA. 2001;286:700-7.

14. Benichou N, Lebbah S, Hajage D, et al. Vascular access for renal replacement therapy among 459 critically ill patients: a pragmatic analysis of the randomized AKIKI trial. Ann Intensive Care. 2021;11:56.

15. Santiago MJ, Sánchez A, López-Herce J, et al. The use of continuous renal replacement therapy in series with extracorporeal membrane oxygenation. Kidney Int. 2009;76:1289-92.

16. Crosswell A, Brain MJ, Roodenburg O. Vascular access site influences circuit life in continuous renal replacement therapy. Crit Care Resusc. 2014;16:127-30.

17. de Tymowski C, Desmard M, Lortat-Jacob B, et al. Impact of connecting continuous renal replacement therapy to the extracorporeal membrane oxygenation circuit. Anaesth Crit Care Pain Med. 2018;37:557-64.

18. Rubin S, Poncet A, Wynckel A, et al. How to perform a haemodialysis using the arterial and venous lines of an extracorporeal life support. Eur J Cardiothorac Surg. 2010;37:967-8.

19. Chuang C-L, Yang W-C. Connecting continuous renal replacement therapy in parallel with extracorporeal membrane oxygenation: is there no problem? Kidney Int. 2010;77:830-0.

20. Michel T, Ksouri H, Schneider AG. Continuous renal replacement therapy: understanding circuit hemodynamics to improve therapy adequacy. Curr Opin Crit Care. 2018;24:455-62.

21. Krivitski N, Galyanov G, Cooper D, et al.: In vitro and in vivo assessment of oxygenator blood volume for the prediction of clot formation in an ECMO circuit (theory and validation). Perfusion 2018; $33: 51-56$.

22. Broman LM, Prahl Wittberg L, et al. Pressure and flow properties of cannulae for extracorporeal membrane oxygenation I: return (arterial) cannulae. Perfusion. 2019;34:58-64.

23. Broman LM, Prahl Wittberg L, Westlund CJ, et al. Pressure and flow properties of cannulae for extracorporeal membrane oxygenation II: drainage (venous) cannulae. Perfusion. 2019;34:65-73.

24. Na SJ, Choi HJ, Chung CR, et al. Using additional pressure control lines when connecting a continuous renal replacement therapy device to an extracorporeal membrane oxygenation circuit. BMC Nephrol. 2018;19:369.

25. de Tymowski C, Augustin P, Houissa H, et al. CRRT Connected to ECMO: Managing High Pressures. ASAIO J. 2017;63:48-52.

26. World Bank Group. International Development, Poverty, \& Sustainability. https://www.worldbank.org/. [Internet]. Available from: https://www.worldbank.org/.

27. Hill JD, O’Brien TG, Murray JJ, et al. Prolonged Extracorporeal Oxygenation for Acute Post-Traumatic Respiratory Failure (Shock-Lung Syndrome): Use of the Bramson Membrane Lung. N Engl J Med. 1972;286:629-34. 
28. Danial P, Hajage D, Nguyen LS, et al. Percutaneous versus surgical femoro-femoral veno-arterial ECMO: a propensity score matched study. Intensive Care Med. 2018;44:2153-61.

29. Shank KR, Profeta E, Wang S, et al. Evaluation of Combined Extracorporeal Life Support and Continuous Renal Replacement Therapy on Hemodynamic Performance and Gaseous Microemboli Handling Ability in a Simulated Neonatal ECLS System. Artif Organs. 2018;42:365-76.

30. Askenazi DJ, Selewski DT, Paden ML, et al. Renal replacement therapy in critically ill patients receiving extracorporeal membrane oxygenation. Clin J Am Soc Nephrol. 2012;7:1328-36.

\section{Tables}


Table 1: Main characteristics of respondents

Characteristics

Overall

(n:298)

Continent

\begin{tabular}{ll}
\hline Europe & $141(47)$ \\
\hline North America & $87(29)$ \\
\hline Asia & $35(12)$ \\
\hline South America & $23(8)$ \\
\hline Oceania & $7(2)$ \\
\hline Africa & $2(1)$ \\
\hline High income country & $249(84)$ \\
\hline Type of critical care unit & \\
\hline Cardiac surgery ICU & $110(37)$ \\
\hline Polyvalent ICU & $74(25)$ \\
\hline Medical ICU & $44(15)$ \\
\hline Surgical ICU & $36(12)$ \\
\hline Trauma center & $17(6)$ \\
\hline Others & $19(6)$ \\
\hline Medical specialty & \\
\hline Medical intensivist & $101(34)$ \\
\hline Anesthesiologist-Intensivist & $97(33)$ \\
\hline Medical physician & $12(4)$ \\
\hline Pediatrician & $34(11)$ \\
\hline Surgeon & $32(11)$ \\
\hline
\end{tabular}

Number of new ECMO cases per month

\begin{tabular}{cc}
\hline $0-3$ & $150(50)$ \\
\hline $4-5$ & $65(22)$ \\
\hline $6-10$ & $32(11)$ \\
\hline $11-20$ & $21(7)$ \\
\hline
\end{tabular}

Page 15/18 
Personal ECMO experience

$\begin{array}{ll}<1 \text { year } & 7(2) \\ 1-5 \text { years } & 46(15) \\ 5-10 \text { years } & 62(21) \\ >10 \text { years } & 100(34)\end{array}$

Table 2: Main fears and actual encountered problems with the DCRE procedure

\begin{tabular}{lccc} 
Fears & \multicolumn{3}{l}{ Problems already encountered } \\
\hline At least one fear & $130(44)$ & At least one problem & $98(33)$ \\
\hline Unstoppable arterial pressure alarm & $50(17)$ & Unstoppable arterial pressure alarm & $58(20)$ \\
\hline Unstoppable venous pressure alarm & $36(12)$ & Unstoppable venous pressure alarm & $44(15)$ \\
\hline Globally stressful & $35(12)$ & Unstoppable TMP alarm & $23(8)$ \\
\hline Unstoppable TMP alarm & $32(11)$ & Air embolism* & $26(9)$ \\
\hline Blood loss & $30(10)$ & Insufficient RRT blood flow & $15(5)$ \\
\hline & & Significant blood loss & $6(2)$ \\
\hline
\end{tabular}

Categorical variables are expressed as $\mathrm{n}(\%)$.

Definition: TMP = Transmembrane pressure; RRT = Renal replacement therapy.

*Including micro air bubbles in ECMO circuit without clinical consequences. 
Table 3: Main reasons for not performing DCRE reported by the DC Group

Reasons

$n=65$

No institutional protocol

$31(48)$

No guidelines

19 (29)

DCRE perceived as unsafe

20 (31)

Difficult management of pressure alarms

No $Y$ connectors on ECMO circuit

Perceived as an inefficient technique

Categorical variables are expressed as $\mathrm{n}(\%)$.

Definition: DCRE = Direct connection of RRT on ECMO; DC = Dialysis catheter; ECMO = Extra corporeal membrane oxygenation; RRT = Renal replacement therapy.

\section{Table 4: Management of DCRE in ECMO group}

$n=100$

Individuals who perform the connections

Perfusionists

Nurses

Type of RRT in case of DCRE (always/often)

CVVHF

CVVHDF

CVVHD

IHD

Choice of RRT type differs when DCRE is performed

Adapting initial RRT settings in case of DCRE

Change RRT or ECMO settings in case of high-pressure alarms

Modify the locations of RRT lines in case of high-pressure alarms

Categorical variables are expressed as numbers and percentages.

Definitions: DCRE = Direct connection of RRT on ECMO; ECMO = Extra corporeal membrane oxygenation; RRT = Renal replacement therapy; IHD = Intermittent hemodialysis; CVVHF = Continuous veno-venous hemofiltration; $\mathrm{CVVHDF}=$ Continuous veno-venous hemodiafiltration; $\mathrm{CVVHD}=$ Continuous veno-venous hemodialysis. 
Figures

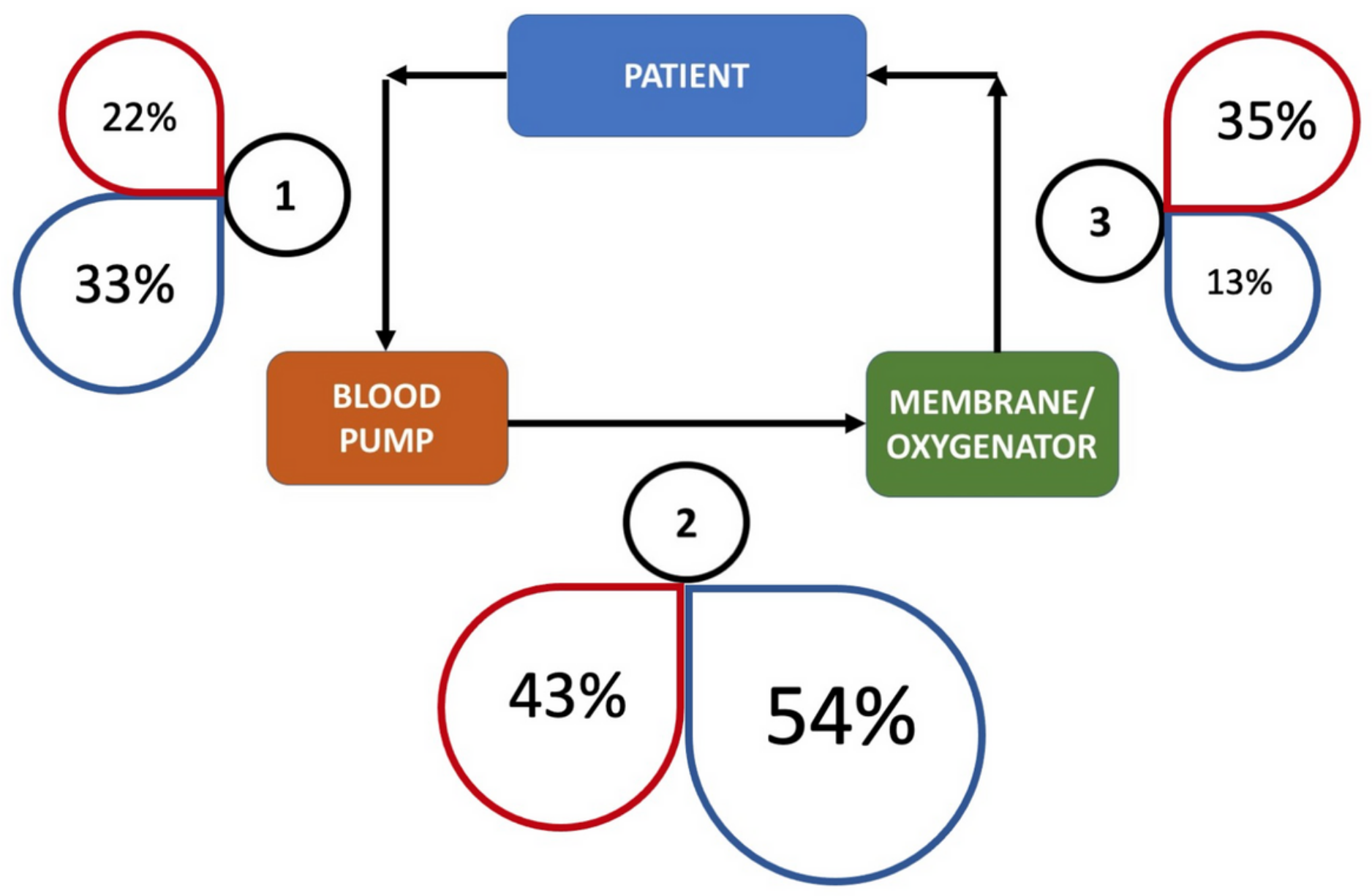

Figure 1

Distribution of the location of RRT line connections on the ECMO circuit for the 100 participants in the ECMO group.

\section{Supplementary Files}

This is a list of supplementary files associated with this preprint. Click to download.

- SupplementalDataFile.doc 